\title{
Mycological and Mycotoxins Analysis of Kareish and Soft Cheese in Assiut, Egypt
}

\author{
A.M. Moharram ${ }^{1, *}$, A. A. Abd El Haleem ${ }^{2}$, R. R. S. Refaie ${ }^{1}$ \\ ${ }^{1}$ Department of Botany and Microbiology, Faculty of Science, Assiut University \\ ${ }^{2}$ Animal Health Research Institute at, Assiut branch \\ *Corresponding author: ahmadmhrrm@yahoo.com
}

Received August 11, 2018; Revised September 12, 2018; Accepted September 26, 2018

\begin{abstract}
During the present study 80 samples of kareish (semi-soft cheese with 20\% fats) and soft cheese (40\% fats) were collected Assiut City during June to December 2015. The fungal content of these samples was evaluated using Dichloran Rose Bengal Chloramphenicol (DRBC) and Yeast Extract Malt extract (YM) agar media. The fungal count per sample ranged from 7 colonies/g in soft cheese to 44800 colonies/g in kareish cheese. the total number of fungal species in kareish cheese was slightly lower on DRBC than on YM (24 and 29 species respectively). The number of fungal species per sample fluctuated between 1-8 species with the highest being recovered from kareish cheese. Aspergillus was the most prevalent genus contaminating $45-85 \%$ of the samples with the highest being recovered from Kareish Cheese. Penicillium came next contaminating 20 - 47.5\% of samples with white soft cheese being the most affected product. Candida, Clavispora, Klyveromyces and Pichia contaminated 2.5-65 \% of samples and the highest incidence was that of Clavispora in kareish cheese. Aspergillus niger, A. fumigatus, A. flavus, Penicillium chrysogenum, $P$. aurantiogriseum and $P$. brevicompactum were the commonest species in cheese samples. Some yeast fungi were identified by sequencing of rRNA gene. Among the 19 species of yeasts Candida tropicalis, Clavispora lusitaniae, Pichia kudriavzevii, Pichia membranifaciens and kluyveromyces lactis were the most common. Testing the natural occurrence of aflatoxins revealed that AFM1 contaminated 55\% and $50 \%$ of kareish and soft cheese respectively whereas AFM2 was found only in $10 \%$ of kareish cheese samples.
\end{abstract}

Keywords: kareish, soft cheese, aflatoxin M1 and M2, mould and yeast fungi

Cite This Article: A.M. Moharram, A. A. Abd El Haleem, and R. R. S. Refaie, "Mycological and Mycotoxins Analysis of Kareish and Soft Cheese in Assiut, Egypt." American Journal of Microbiological Research, vol. 6, no. 4 (2018): 165-172. doi: 10.12691/ajmr-6-4-4.

\section{Introduction}

A wide variety of milk products including cheese, yoghurt, butter and ice cream are manufactured from milk of cow, buffaloes, goats, sheep and camels [1]. Cheese is made from fermented raw milk and is consumed as one of the highly nutritious dairy products in several countries. It is a good source of proteins, fats, vitamins and minerals such as calcium, magnesium and phosphorus [2].

Kareish cheese is a local type of fresh cheese in Egypt. It is preferred by Egyptian consumers due to its high protein content and low price [3]. Kareish cheese is made from skim cow or buffalo's milk which is extracted directly into special earthenware pots and kept without disturbance to allow the fat to rise to the surface forming a cream layer. The cream layer is then removed, and the curd is poured onto a mat which is tied and hung for few days to allow the drainage of the whey. The cheese is then cut into suitable pieces with the addition of some salt. The cheese is left for few hours in the mat till whey no longer drains out, then it is ready to be consumed as fresh soft cheese containing about $20 \%$ fats. The second type of cheese is produced by some dairy companies in Egypt with $40 \%$ fat content. Because milk and milk products are rich in moisture and several nutrients they are subjected to microbial contaminated which grow and multiply causing spoilage and economic losses to the dairy industry [4]. The contamination of milk products with different types of fungi particularly of species of Aspergillus, Fusarium and Penicillium constitute a public health hazard as these fungi are known to produce mycotoxins that are injurious to human health [5]. Some of the common yeasts related to dairy products are: Debaryomyces hansenii, Galactomyces geotrichum, Yarrowia lipolytica, Kluyveromyce marxianus, K. lactis var. lactis, and Saccharomyces cerevisiae [6].

Aflatoxins are natural compounds produced by Aspergillus flavus and A. parasiticus. Aflatoxins include B1, B2, G1, and G2. B1 is usually found at high concentration in contaminated food and feedstuffs [7]. Aflatoxins M1 and M2 are derivatives of B1 and B2, which are excreted in the milk of animals [8]. 
This investigation was carried out to study the prevalence and density of filamentous and yeast fungi in two types of cheese and to evaluate the natural occurrence of mycotoxins in these products.

\section{Materials and Methods}

\subsection{Collection of Samples}

Sample of kareish and soft cheese (40 samples for each) were randomly collected from different local markets in Assiut City, Egypt during the period from June to December 2015. Samples were kept at $-20^{\circ} \mathrm{C}$ till mycological and toxicological analysis.

\subsection{Mycological Analysis}

The dilution plate technique was employed to isolate fungi contaminating kareish and soft cheese using dichloran rosebengal chloramphenicol (DRBC) and yeast extract-malt extract (YM) agar [9]. Cultures were incubated at $28 \pm 2^{\circ} \mathrm{C}$ for $7-10$ days after which the developing fungal colonies were counted, identified and preserved.

\subsection{Identification of Fungal Isolates}

The following references were used for phenotypic identification [9,10,11,12]. Molecular characterization of some yeast isolates was done with the help of Solgent Company, Daejeon South Korea [13].

\subsection{Extraction and Detection of Mycotoxins}

This part was performed with the help of food analysis center, Faculty of Veterinary Medicine, Benha University according to the following protocol:

2.4.1 Ten grams of each cheese sample was blended with $100 \mathrm{ml}$ extraction solvent: acetone: water $(85: 15, \mathrm{v} / \mathrm{v})$ for $30 \mathrm{~min}$. Then the mixture was filtered through Whatman No. 1 filter paper. After filtration, the extract (5 ml) was diluted with water $(75 \mathrm{ml})$.

2.4.2 High performance liquid chromatography (HPLC) used for aflatoxin determination was an Agilent 1100 HPLC system, Agilen Technologies, Waldbronn, Germany, equipped with quaternary pump model G 1311A, UV detector (Model G 1314A) set at 254nm wavelength. Also, auto sampler (model G1329A VP-ODS) and Shim pack $(150 \times 4.6 \mathrm{~mm})$ column (Shimadzu, Kyoto, Japan) were used.

2.4.3 The stock standard solutions of AFB1, AFB2, AFM1 and AFM2 were prepared by dissolving the solid standard in benzene: acetonitrile (98:2, v/v).

2.4.4 The European Commission was used as for guidelines and criteria to assess the method validation. Selectivity was determined from Retention time, ion ratios, and identification-points (IP) for each analyte. Calibration standards were prepared by combining standard solutions into the solvent and blank matrix extracts (matrix-matching) to yield the desired concentrations in the range of $10-500 \mu \mathrm{g} / \mathrm{L}$ for each analyte.

\section{Results and Discussion}

\subsection{Fungi Isolated from Kareish Cheese}

Thirty-one species belonging to 21 genera were isolated from kareish cheese samples on both DRBC (23 species) and YM (27 species)

\subsubsection{On DRBC medium}

With reference to Table 1 and Table 2, the total number of fungal colonies per sample ranged from 1520-44800 colonies / g of kareish cheese. The number of species per sample ranged from 2- 8 species with the richest samples being No. 81 and No. 93.

The most common genera were Aspergillus, Clavispora and Pichia being isolated from $85 \%, 60 \%$ and $50 \%$ of samples respectively. The percentage count of Clavispora (26.28\%) was markedly higher than that of Aspergillus and Pichia $(7.10 \%$, and $13.27 \%$ of the gross total population of fungi respectively). Clavispora lusitanie was the only representative species of its genus.

Pichia was represented by 4 species of which $P$. kudriavzevii was of moderate incidence $(32.5 \%$ of total samples matching $10.7 \%$ of total count of fungi). P. anomala, P. cactophila and P. membranifacenss occurred in low incidence. Candida tropicalis, Galactomyces candida and Magnsiomyces sp. were isolated from 40\%, $40 \%$ and $27.5 \%$ of kareish cheese samples (moderate incidence) accounting for $13.71 \%, 14.1 \%$ and $8.18 \%$ of total fungal population respectively. Also, Kluyveromyces contaminated $30 \%$ of kareish cheese samples accounting for $8.55 \%$ of total fungal population. Kluyveromyces was represented by 2 species namely $K$. lactis and $K$. marxianus (25\% and $7.5 \%$ of cheese samples respectively).

\subsubsection{OnYM Medium}

With reference to Table 1 \& Table 2, the total number of fungal colonies per sample ranged from 1680-43200 colonies / g. The number of species ranged from (2 - 6 species / sample). Aspergillus, Pichia and Clavispora were the most common genera being isolated from $67.5 \%$, $52.5 \%$ and $65 \%$ of kareish cheese samples respectively. The percentage counts of Pichia and Clavispora (22.66\% and $23.27 \%$ respectively) were much higher than that of Aspergillus 5.19\% of the gross total population of fungi. Aspergillus was represented by 4 species of which A. flavus and $A$. niger occurred in $30 \%$ and $42.5 \%$ of samples matching $2.88 \%$ and $2.07 \%$ of total count of fungi respectively. Four species of Pichia were recovered of which $P$. kudriavzevii occurred in moderate incidence (27.5\% of the total samples) matching $14.35 \%$ of total count of fungi. Each of Clavispora and Galactomyces was represented by one species (C. lusitanii and Galactomyces candida which occurred in high and moderate incidences $65 \%$ and $37.5 \%$ of samples matching 22.66 and $11.14 \%$ of total count of fungi respectively.

Most of the moulds that contaminate kareish cheese in this work especially those belonging to Aspergillus, Penicillium, Cladosporium, Mucor and Fusarium were also isolated by several investigators [14,15,16,17,18]. Similarly the major yeasts contaminating kareish cheese 
especially Candida lipolotica (= Yarowiiai lipolotica), C. parapsiliopsis C. catenulata, C. tropicalis and C. zeylanoides, Kluyveromyces lactis and K. Marxianus, Rhodotorula sp., Pichia sp., Cryptococcus sp. and Galactomyces candida were recovered from similar samples [14,15,16,18,19,20].

\subsubsection{Phylogeny of Yeasts Isolated from Kareish Cheese}

With reference to Fig. (1), the phylogenic tree covered eight different yeast species. Identification of each species was done on the basis of its high similarity with reference strains in the Gene Bank. Two species of Pichia were identified, namely Pichia kudriavzevii and Pichia anomala. The remaining species included Galactomyces candidum (anamorph=Geotrichum candidum), Candida tropicalis, Clavispora lusitaniae, Trichosporon asahii, Lecythophora sp. and Kluyveromyces lactis.

\subsection{Fungi Isolated from Soft Cheese}

A total of 38 species belonging to 22 genera were recovered from the 40 soft cheese samples using DRBC (17 genera and 29 species) and YM (17 genera and 30 species) as shown in Table $1 \&$ Table 2 . Three out of the 40 samples were free of fungal propagules on both DRBC and YM media.

\subsubsection{On DRBC Medium}

The total number of fungal colonies per sample ranged from 6.7-3153.02 colonies / g of soft cheese (Table 1 \& Table 2). Seven (17.5\%) of white cheese samples were completely free of fungi. The number of species ranged from (1 - 6 species / sample) .

Penicillium was isolated from 50\% samples matching $29.5 \%$ of total population of fungi. P. chrysogenum occurred in moderate incidence contaminating 30\% of white cheese samples accounting for $8.43 \%$ of the total fungal population. Aspergillus and Clavispora occurred in modrate incidence contaminating $47 \%$ and $37.5 \%$ of white cheese samples respectively. The precentage count of Clavispora markedly exceeded that of Aspergillus (33.3\% and 9.21\%respectively). Clavispora was represented only by one species (Clavispora lusitanii). Aspergillus was represented by 6 species of which $A$. niger occurred in $25 \%$ of samples accounting for $2.19 \%$ of the total fungal population.

\subsubsection{On YM Medium}

In contaminated samples the total number of fungal colonies per sample ranged from 6.6-6120 colonies/g (Table 1 \& Table 2). Four samples (10\%) were completely free of fungi. The number of species ranged from (1 - 8 species / sample). Aspergillus, Penicillium, Clavispora and Pichia occurred in modrate incidence and were isolated from $45 \%, 47.5 \%, 35 \%$ and $17.5 \%$ of white cheese samples respectively. The count of Clavispora (27.3\%) markedly exceeded that of Aspergillus, Penicillium and Pichia7.80\%, $18.24 \%$ and $9.07 \%$ of the gross total fungal population respectively.

Among the 5 species of Aspergillus, A. fumigatus was contaminating $32.5 \%$ of soft cheese samples accounting for $3.78 \%$ of the total fungal population. Eight species of Penicillium were identified from which $P$. chrysogenum appeared in $30 \%$ of samples representing $7.35 \%$ of the total fungal count. Clavispora lusitanii, Pichia kudriavzvii, $P$. parapsiliopses and $P$. membranifacens were the representative species of corresponding genera.

A lot of Egyptian investigators analyzed soft cheese for yeast and mould contamination among them Ibrahim et al. (2015) [21], Sayed et al. (2011) [22] who reported that yeasts and moulds contaminated $100 \%$ of tested white soft cheese samples while, ELbagory et al. (2014) [23] and Hamms (2014) [16] found yeasts in $77.1 \%$ and $25 \%$ and molds in $94.3 \%$ and $20 \%$ of samples respectively. Differences in the count of fungal propagules may be due to the geographical location of producing companies, duration of storage and the hygienic status of persons dealing with these products. Lund et al. (1995) analyzed soft cheese from Denmark and isolated Penicillium auarantiogriseum and P. chrysogenum [24]. Johnson (2001) isolated Geotrichum candidum, Kluyveromyces marxians, Pichia sp and Candida sp. from cheese [25]. Torkar and Teger (2006) from Slovenia reported that $60 \%$ of tested cheese samples were contaminated with yeasts and moulds which included Aspergillus sp., Penicillium sp., Geotrichum candidum and Moniliella spathulate [26]. Lavoie et al. (2012) analyzed cheese samples from Quebec and isolated Candida (C. catenulata, C. parapsiliopsis, C. parragosa, C. tropicalis, C. zeylanoides), Cryptococcus curvatus, Pichia (P. kudriavzevii, P. mempranifacens), Rhodotrola mucilaginosa, Trichosporon asahii, Trichosporon jirovece, Kluyveromyces marxianus, A. fumigatus, C. cladosporioides, Erotium sp and Mucor circinelloides [27]. Allaraj et al. (2013) [28] and Khalifa et al. (2013) [5] isolated Aspergillus flavus, A. niger, Penicillium chrysogenum, Mucor sp., Cladosporium sp., and Fusarium sp. from cheese while Banjara et al. (2015) obtained some yeasts including (Geotrichum candidum, Kluyveromyces lactis, Pichia kudriavzevii, C.parapsiliopsis) [29]. Also Aspergillus sp., Penicillium sp., Mucour sp., Cladosporium sp., Rhizopus sp., Scopulariopsis sp., Candida sp., Rhodotorula sp were isolated from cheese by ELbagory et al. (2014) [23] and Hameed (2016) [17]. While Chipilev et al. (2016) isolated these moulds from cheese in addition to Candida sp. and Rhodotrola sp. at percentages of $70 \%$ for molds and $63.3 \%$ for yeasts [30].

It was very interesting to observe that the total fungal load contaminating kareish cheese was much higher than that in soft cheese (42 folds) as shown on DRBC medium. Nearly the same observation was recorded in case of YM medium whereas the gross fungal population obtained from kareish cheese was 26.3 folds that of soft cheese. A plausible explanation of the reduced number of fungal propagules in soft cheese is the addition of relatively high amount of $\mathrm{NaCl}$ salt which reduce the vegetative growth of several molds and yeasts. Also, thermal treatment of milk during pasteurization often contributes to eliminate many of the contaminating fungi.

The heavy contamination of kareish cheese by molds an and yeasts is often attributed to exposure of milk to air mycobiota during processing, transport and marketing of this kind of cheese. Most of kareish cheese is made by dairy farmers, who often don't follow the correct higyenic measures in their work. 
Table 1. Counts (cfu/g) and number of species of fungi isolated from kareish and soft cheese on DRBC and YM media

\begin{tabular}{|c|c|c|c|c|c|c|c|c|c|}
\hline \multicolumn{5}{|c|}{ Kareish cheese } & \multicolumn{5}{|c|}{ Soft cheese } \\
\hline \multirow[b]{2}{*}{$\begin{array}{c}\text { Sample } \\
\text { No. }\end{array}$} & \multicolumn{2}{|c|}{ DRBC } & \multicolumn{2}{|c|}{ YM } & \multirow[b]{2}{*}{$\begin{array}{c}\text { Sample } \\
\text { No. }\end{array}$} & \multicolumn{2}{|c|}{ DRBC } & \multicolumn{2}{|c|}{ YM } \\
\hline & $\begin{array}{c}\text { Fungal } \\
\text { count }\end{array}$ & $\begin{array}{l}\text { No. of } \\
\text { species }\end{array}$ & $\begin{array}{c}\text { Fungal } \\
\text { count }\end{array}$ & $\begin{array}{l}\text { No. of } \\
\text { species }\end{array}$ & & $\begin{array}{c}\text { Fungal } \\
\text { count }\end{array}$ & $\begin{array}{l}\text { No. of } \\
\text { species }\end{array}$ & $\begin{array}{c}\text { Fungal } \\
\text { count }\end{array}$ & $\begin{array}{c}\text { No. of } \\
\text { species }\end{array}$ \\
\hline 1 & 3760 & 8 & 2820 & 4 & 41 & 520 & 3 & 880 & 3 \\
\hline 2 & 1520 & 4 & 1680 & 4 & 42 & 0 & 0 & 4280 & 5 \\
\hline 3 & 7600 & 5 & 6980 & 5 & 43 & 100 & 2 & 40 & 1 \\
\hline 4 & 3500 & 5 & 3440 & 5 & 44 & 240 & 3 & 380 & 3 \\
\hline 5 & 8160 & 6 & 7680 & 5 & 45 & 520 & 4 & 940 & 3 \\
\hline 6 & 3940 & 4 & 3540 & 4 & 46 & 1960 & 3 & 6120 & 6 \\
\hline 7 & 8680 & 3 & 8560 & 3 & 47 & 200 & 2 & 40 & 1 \\
\hline 8 & 9360 & 5 & 8920 & 4 & 48 & 360 & 3 & 80 & 1 \\
\hline 9 & 9520 & 5 & 9080 & 4 & 49 & 360 & 3 & 300 & 3 \\
\hline 10 & 9680 & 4 & 9000 & 3 & 50 & 860 & 5 & 846.6 & 5 \\
\hline 11 & 6880 & 5 & 8760 & 3 & 51 & 1093 & 4 & 779.9 & 2 \\
\hline 12 & 9120 & 4 & 10360 & 6 & 52 & 220 & 2 & 213.3 & 3 \\
\hline 13 & 9000 & 8 & 7520 & 3 & 53 & 0 & 0 & 0 & 0 \\
\hline 14 & 20480 & 6 & 15360 & 2 & 54 & 700 & 2 & 100 & 2 \\
\hline 15 & 15440 & 4 & 17200 & 2 & 55 & 2160 & 5 & 2440 & 5 \\
\hline 16 & 9200 & 3 & 13200 & 3 & 56 & 1400 & 6 & 1900 & 5 \\
\hline 17 & 18960 & 6 & 18480 & 5 & 57 & 0 & 0 & 93.3 & 1 \\
\hline 18 & 18880 & 5 & 19200 & 3 & 58 & 427 & 5 & 453.3 & 2 \\
\hline 19 & 20000 & 2 & 23120 & 4 & 59 & 367 & 2 & 619.9 & 2 \\
\hline 20 & 22560 & 4 & 24240 & 4 & 60 & 0 & 0 & 60 & 2 \\
\hline 21 & 18240 & 3 & 18480 & 3 & 61 & 0 & 0 & 0 & 0 \\
\hline 22 & 18320 & 3 & 19760 & 3 & 62 & 173 & 3 & 333.3 & 4 \\
\hline 23 & 15040 & 3 & 15120 & 5 & 63 & 213 & 2 & 126.7 & 2 \\
\hline 24 & 39040 & 5 & 43200 & 3 & 64 & 1680 & 6 & 1480 & 5 \\
\hline 25 & 40960 & 5 & 37760 & 5 & 65 & 1740 & 4 & 2760 & 8 \\
\hline 26 & 30880 & 4 & 28320 & 3 & 66 & 7 & 1 & 186.7 & 2 \\
\hline 27 & 32800 & 4 & 23680 & 5 & 67 & 0 & 0 & 0 & 0 \\
\hline 28 & 11360 & 4 & 16960 & 4 & 68 & 33.3 & 3 & 100 & 4 \\
\hline 29 & 32160 & 6 & 33440 & 6 & 69 & 60 & 2 & 93.3 & 3 \\
\hline 30 & 37920 & 2 & 32480 & 6 & 70 & 0 & 0 & 110 & 5 \\
\hline 31 & 24160 & 4 & 24640 & 3 & 71 & 3153 & 2 & 2499.8 & 3 \\
\hline 32 & 27680 & 6 & 23200 & 5 & 72 & 10 & 1 & 10 & 1 \\
\hline 33 & 22720 & 4 & 23360 & 5 & 73 & 13.3 & 2 & 13.3 & 2 \\
\hline 34 & 41600 & 6 & 30080 & 4 & 74 & 147 & 4 & 40 & 2 \\
\hline 35 & 44800 & 3 & 33920 & 2 & 75 & 13.3 & 2 & 6.6 & 1 \\
\hline 36 & 34880 & 3 & 35840 & 5 & 76 & 6.7 & 1 & 0 & 0 \\
\hline 37 & 32640 & 3 & 30560 & 3 & 77 & 27 & 2 & 60 & 2 \\
\hline 38 & 9760 & 4 & 8160 & 4 & 78 & 40 & 2 & 93.3 & 3 \\
\hline 39 & 24480 & 5 & 23200 & 5 & 79 & 27 & 2 & 73.3 & 4 \\
\hline 40 & 37600 & 4 & 32480 & 4 & 80 & 67 & 2 & 106.7 & 1 \\
\hline
\end{tabular}


Table 2. Total counts (TC as colonies/g in all samples) and frequencies of occurrence (F) of fungal genera and species isolated from kareish and soft cheese

\begin{tabular}{|c|c|c|c|c|c|c|c|c|c|c|c|c|}
\hline \multirow{3}{*}{ Genera and Species } & \multicolumn{6}{|c|}{ Kareish cheese } & \multicolumn{6}{|c|}{ Soft cheese } \\
\hline & \multicolumn{3}{|c|}{ DRBC } & \multicolumn{3}{|c|}{ YM } & \multicolumn{3}{|c|}{ DRBC } & \multicolumn{3}{|c|}{ YM } \\
\hline & \%TC & F\&OR & $\% \mathbf{F}$ & $\%$ TC & F\&OR & $\% \mathrm{~F}$ & \%TC & F\&OR & $\% \mathrm{~F}$ & $\% \mathrm{TC}$ & F\&OR & $\% F$ \\
\hline Alternaria alternata & 0 & 0 & 0 & 0 & 0 & 0 & 0 & 0 & 0 & 0.42 & $1 \mathrm{~L}$ & 2.5 \\
\hline Aspergillus & 7.10 & $34 \mathrm{H}$ & 85 & 5.19 & $27 \mathrm{H}$ & 67.5 & 9.21 & $19 \mathrm{M}$ & 47.5 & 7.80 & $18 \mathrm{M}$ & 45 \\
\hline A. carneus & 0 & 0 & 0 & 0 & 0 & 0 & 0.21 & $2 \mathrm{~L}$ & 5 & 0 & 0 & 0 \\
\hline A. flavus & 2.9 & $20 \mathrm{M}$ & 50 & 3 & $14 \mathrm{M}$ & 35 & 2.22 & $8 \mathrm{~L}$ & 17.5 & 1.84 & $5 \mathrm{~L}$ & 12.5 \\
\hline A. fumigatus & 1.31 & $10 \mathrm{M}$ & 25 & 0.05 & $3 \mathrm{~L}$ & 7.5 & 3.42 & $9 \mathrm{~L}$ & 22.5 & 3.78 & $13 \mathrm{M}$ & 32.5 \\
\hline A. nidulans & 0.05 & $1 \mathrm{~L}$ & 2.5 & 0.04 & $1 \mathrm{~L}$ & 2.5 & 0.07 & $1 \mathrm{~L}$ & 2.5 & 0.02 & $1 \mathrm{~L}$ & 2.5 \\
\hline A. niger & 2.83 & $16 \mathrm{M}$ & 40 & 2.07 & $17 \mathrm{M}$ & 42.5 & 2.19 & $10 \mathrm{M}$ & 25 & 1.67 & $7 \mathrm{~L}$ & 17.5 \\
\hline A. sulphureus & 0 & 0 & 0 & 0 & 0 & 0 & 1.16 & $1 \mathrm{~L}$ & 2.5 & 0 & 0 & 0 \\
\hline A. sydowii & 0 & 0 & 0 & 0 & 0 & 0 & 0 & 0 & 0 & 0.02 & $1 \mathrm{~L}$ & 2.5 \\
\hline A. terreus & 0 & 0 & 0 & 0.07 & $1 \mathrm{~L}$ & 2.5 & 0 & 0 & 0 & 0.49 & $1 \mathrm{~L}$ & 2.5 \\
\hline Chaetomium globosum & 0.04 & $1 \mathrm{~L}$ & 2.5 & 0 & 0 & 0 & 0.11 & $1 \mathrm{~L}$ & 2.5 & 0 & 0 & 0 \\
\hline C. cladosporioides & 0.01 & $1 \mathrm{~L}$ & 2.5 & 0 & 0 & 0 & 0.07 & $2 \mathrm{~L}$ & 5 & 0.14 & $1 \mathrm{~L}$ & 2.5 \\
\hline Fusarium & 0.03 & $1 \mathrm{~L}$ & 2.5 & 0.04 & $1 \mathrm{~L}$ & 2.5 & 0.04 & $1 \mathrm{~L}$ & 2.5 & 0 & 0 & 0 \\
\hline F. oxysporum & 0 & 0 & 0 & 0.04 & $1 \mathrm{~L}$ & 2.5 & 0 & 0 & 0 & 0 & 0 & 0 \\
\hline F. Solani & 0 & 0 & 0 & 0 & 0 & 0 & 0.04 & $1 \mathrm{~L}$ & 2.5 & 0 & 0 & 0 \\
\hline F. Verticillioides & 0.03 & $1 \mathrm{~L}$ & 2.5 & 0 & 0 & 0 & 0 & 0 & 0 & 0 & 0 & 0 \\
\hline Mucor himalis & 0 & 0 & 0 & 0.04 & $1 \mathrm{~L}$ & 2.5 & 0 & 0 & 0 & 0.56 & $1 \mathrm{~L}$ & 2.5 \\
\hline Paeciiomyces sp. & 0 & 0 & 0 & 0 & 0 & 0 & 0 & 0 & 0 & 0.56 & $1 \mathrm{~L}$ & 2.5 \\
\hline Penicillium & 3.14 & $9 \mathrm{~L}$ & 22.5 & 3.85 & 8 & 20 & 29.51 & $20 \mathrm{H}$ & 50 & 18.24 & $19 \mathrm{M}$ & 47.5 \\
\hline P.aurantiogriseum & 0.61 & $5 \mathrm{~L}$ & 7.5 & 0.99 & $4 \mathrm{~L}$ & 10 & 16.9 & $4 \mathrm{~L}$ & 10 & 8.32 & $3 \mathrm{~L}$ & 7.5 \\
\hline P. brevicompactum & 0.24 & $1 \mathrm{~L}$ & 2.5 & 0.25 & $1 \mathrm{~L}$ & 2.5 & 0.42 & $1 \mathrm{~L}$ & 2.5 & 0.02 & $1 \mathrm{~L}$ & 2.5 \\
\hline P. chrysogenum & 1.81 & $9 \mathrm{~L}$ & 22.5 & 2.1 & $6 \mathrm{~L}$ & 15 & 8.43 & $12 \mathrm{M}$ & 30 & 7.35 & $12 \mathrm{M}$ & 30 \\
\hline P. citrinum & 0 & 0 & 0 & 0 & 0 & 0 & 2.15 & $4 \mathrm{~L}$ & 10 & 0.33 & $1 \mathrm{~L}$ & 2.5 \\
\hline P.duclauxii & 0.04 & $1 \mathrm{~L}$ & 2.5 & 0 & 0 & 0 & 0 & 0 & 0 & 0 & 0 & 0 \\
\hline P. griseofulvum & 0 & 0 & 0 & 0 & 0 & 0 & 0.12 & $2 \mathrm{~L}$ & 5 & 0.8 & $2 \mathrm{~L}$ & 5 \\
\hline P.oxalicum & 0 & 0 & 0 & 0 & 0 & 0 & 0 & 0 & 0 & 0.23 & $1 \mathrm{~L}$ & 2.5 \\
\hline P. pinophilum & 0.44 & $1 \mathrm{~L}$ & 2.5 & 0.51 & $1 \mathrm{~L}$ & 2.5 & 0 & 0 & 0 & 0.07 & $1 \mathrm{~L}$ & 2.5 \\
\hline P. purprogenum & 0 & 0 & 0 & 0 & 0 & 0 & 1.48 & $1 \mathrm{~L}$ & 2.5 & 1.12 & $1 \mathrm{~L}$ & 2.5 \\
\hline R. stolonifer & 0 & 0 & 0 & 0.04 & $1 \mathrm{~L}$ & 2.5 & 0 & 0 & 0 & 0.42 & $2 \mathrm{~L}$ & 5 \\
\hline Scopulariopsis & 0 & 0 & 0 & 0.02 & $1 \mathrm{~L}$ & 2.5 & 0.04 & $1 \mathrm{~L}$ & 2.5 & 0.22 & $3 \mathrm{~L}$ & 7.5 \\
\hline Scopulariopsis bromptii & 0 & 0 & 0 & 0.02 & $1 \mathrm{~L}$ & 2.5 & 0.04 & $1 \mathrm{~L}$ & 2.5 & 0.22 & $3 \mathrm{~L}$ & 7.5 \\
\hline Setosphaeria rostrata & 0 & 0 & 0 & 0 & 0 & 0 & 0.64 & $1 \mathrm{~L}$ & 2.5 & 0 & 0 & 0 \\
\hline Synspholastrum racemosum & 0 & 0 & 0 & 0.07 & $1 \mathrm{~L}$ & 2.5 & 0 & 0 & 0 & 0 & 0 & 0 \\
\hline Black yeasts & 0 & 0 & 0 & 0.16 & $3 \mathrm{~L}$ & 7.5 & 0 & 0 & 0 & 0 & 0 & 0 \\
\hline Candida & 13.71 & $16 \mathrm{M}$ & 40 & 8.2 & $10 \mathrm{~L}$ & 25 & 3.70 & $3 \mathrm{~L}$ & 7.5 & 13.64 & $6 \mathrm{~L}$ & 15 \\
\hline C.parapsiliopsis & 0 & 0 & 0 & 0 & 0 & 0 & 0.21 & $1 \mathrm{~L}$ & 2.5 & 1.64 & $3 \mathrm{~L}$ & 7.5 \\
\hline C. tropicalis & 13.71 & $16 \mathrm{M}$ & 40 & 8.2 & $10 \mathrm{~L}$ & 25 & 3.49 & $2 \mathrm{~L}$ & 5 & 12 & $4 \mathrm{~L}$ & 10 \\
\hline Clavispora lusitaniae & 26.28 & $24 \mathrm{H}$ & 60 & 23.27 & $26 \mathrm{H}$ & 65 & 33.3 & $15 \mathrm{M}$ & 37.5 & 27.3 & $14 \mathrm{M}$ & 35 \\
\hline Galactomyces candidas & 14.1 & $16 \mathrm{M}$ & 40 & 11.14 & $15 \mathrm{M}$ & 37.5 & 2.96 & $1 \mathrm{~L}$ & 2.5 & 3.7 & $1 \mathrm{~L}$ & 2.5 \\
\hline Kluyveromyces & 8.55 & $12 \mathrm{M}$ & 30 & 8.84 & $10 \mathrm{~L}$ & 25 & 2.65 & $1 \mathrm{~L}$ & 2.5 & 6.77 & $5 \mathrm{~L}$ & 12.5 \\
\hline Kluyveromyces lactis & 8.22 & $10 \mathrm{M}$ & 25 & 8.36 & $9 \mathrm{~L}$ & 22.5 & 2.65 & $1 \mathrm{~L}$ & 2.5 & 6.77 & $5 \mathrm{~L}$ & 12.5 \\
\hline Kluyveromyces marxians & 0.34 & $3 \mathrm{~L}$ & 7.5 & 0.47 & $1 \mathrm{~L}$ & 2.5 & 0 & 0 & 0 & 0 & 0 & 0 \\
\hline Lecythophora sp. & 1.77 & $3 \mathrm{~L}$ & 7.5 & 2.13 & $4 \mathrm{~L}$ & 10 & 0 & 0 & 0 & 0 & 0 & 0 \\
\hline Magnisiomyces capitatus & 8.18 & $11 \mathrm{M}$ & 27.5 & 5.95 & $7 \mathrm{~L}$ & 17.5 & 0 & 0 & 0 & 2.09 & $2 \mathrm{~L}$ & 5 \\
\hline Moniliella spathulata & 0 & 0 & 0 & 0 & 0 & 0 & 1.87 & $1 \mathrm{~L}$ & 2.5 & 2.51 & $2 \mathrm{~L}$ & 5 \\
\hline Pichia & 13.27 & $20 \mathrm{H}$ & 50 & 22.66 & $21 \mathrm{H}$ & 52.5 & 11.54 & $10 \mathrm{~L}$ & 25 & 9.07 & $10 \mathrm{M}$ & 25 \\
\hline P. anomela & 1.61 & $6 \mathrm{~L}$ & 15 & 2.13 & $5 \mathrm{~L}$ & 12.5 & 0 & 0 & 0 & 0 & 0 & 0 \\
\hline P. cactophila & 1.95 & $6 \mathrm{~L}$ & 15 & 7.94 & $7 \mathrm{~L}$ & 17.5 & 0.21 & $1 \mathrm{~L}$ & 2.5 & 0 & 0 & 0 \\
\hline P. kudriavzevii & 10.7 & $13 \mathrm{M}$ & 32.5 & 14.35 & $11 \mathrm{M}$ & 27.5 & 12.17 & $6 \mathrm{~L}$ & 10 & 7.88 & $6 \mathrm{~L}$ & 15 \\
\hline P.manshurica & 0 & 0 & 0 & 0 & 0 & 0 & 1.87 & $1 \mathrm{~L}$ & 2.5 & 0 & 0 & 0 \\
\hline P. membranifaciens & 2.64 & $4 \mathrm{~L}$ & 10 & 1.55 & $2 \mathrm{~L}$ & 5 & 0.67 & $4 \mathrm{~L}$ & 10 & 1.12 & $4 \mathrm{~L}$ & 10 \\
\hline P. parapsiliopses & 0 & 0 & 0 & 0 & 0 & 0 & 0 & 0 & 0 & 0.07 & $1 \mathrm{~L}$ & 2.5 \\
\hline Rhodotrola mucilaginosa & 0 & 0 & 0 & 0.53 & $1 \mathrm{~L}$ & 2.5 & 0.28 & $1 \mathrm{~L}$ & 2.5 & 0 & 0 & 0 \\
\hline Trichosporon & 0.11 & $1 \mathrm{~L}$ & 2.5 & 0.76 & $2 \mathrm{~L}$ & 5 & 0 & 0 & 0 & 0.42 & $2 \mathrm{~L}$ & 5 \\
\hline Trichosporon asahii & 0.11 & $1 \mathrm{~L}$ & 2.5 & 0.76 & $2 \mathrm{~L}$ & 5 & 0 & 0 & 0 & 0 & 0 & 00 \\
\hline Trichosporon insectroum & 0 & 0 & 0 & 0 & 0 & 0 & 0 & 0 & 0 & 0.42 & $2 \mathrm{~L}$ & 5 \\
\hline Yarowiilipolotica & 0 & 0 & 0 & 3.74 & $2 \mathrm{~L}$ & 5 & 0.53 & $1 \mathrm{~L}$ & 2.5 & 6.68 & $5 \mathrm{~L}$ & 12.5 \\
\hline Total count & \multicolumn{3}{|c|}{793280} & \multicolumn{3}{|c|}{753780} & \multicolumn{3}{|c|}{18895.99} & \multicolumn{3}{|c|}{28659.28} \\
\hline NO of genera 33 & & 14 & & & $18+1$ & & & 17 & & & 17 & \\
\hline NO of species $66(63+3)+1$ & & $23+1+1$ & & & $27+1+2$ & & & $29+2$ & & & $30+1$ & \\
\hline
\end{tabular}

OR = Occurrence Remarks: H = High Occurrence, 10-20 cases; $\mathrm{M}$ = Moderate Occurrence, 5-9 cases, L = Low Occurrence, 1- 4 cases. 


\subsubsection{Phylogeny of Yeasts Isolated from Soft Cheese}

Performing 18S rDNA sequencing allowed the molecular identification of 10 different yeast species (Figure 2). Identification of each species was based on its close relationship with reference strains in the Gene bank. Three species of Pichia were identified, namely
P. membranifaciens, $P$. manshurica and P. cactophila. Also, two species of Candida were identified (C. tropicalies and C. parapsiliosis). The remaining species including Yarrowia lipolytica, Rhodotorula mucilaginosa, Clavispora lusitanii, Trichosporon insectorum and Moniliella spathulata.

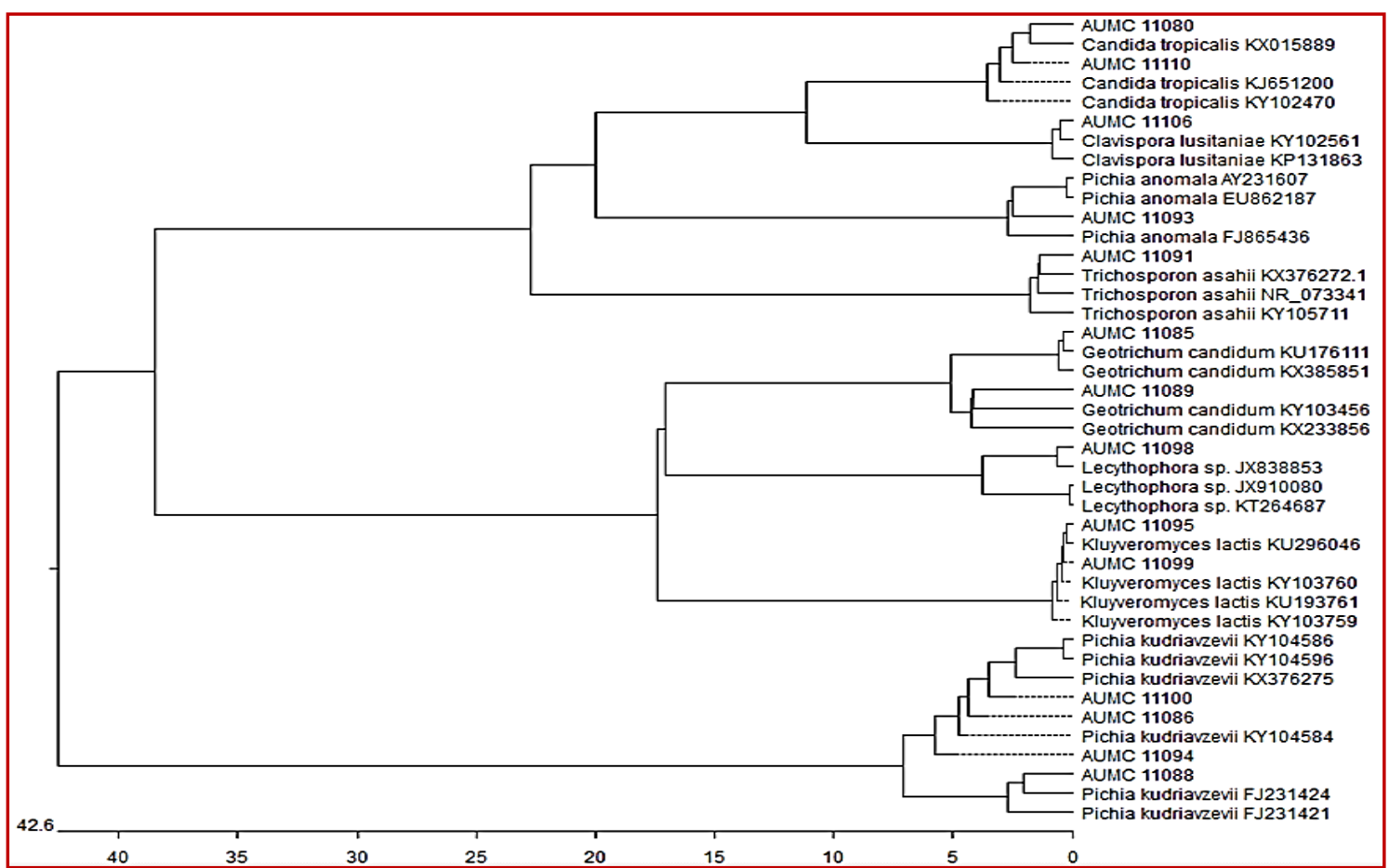

Figure 1. Phylogenetic tree for fungal species isolated from kareish cheese (given AUMC numbers). Reference strains of corresponding fungi are involved in the tree (given CBS, ATCC or AUMC numbers)

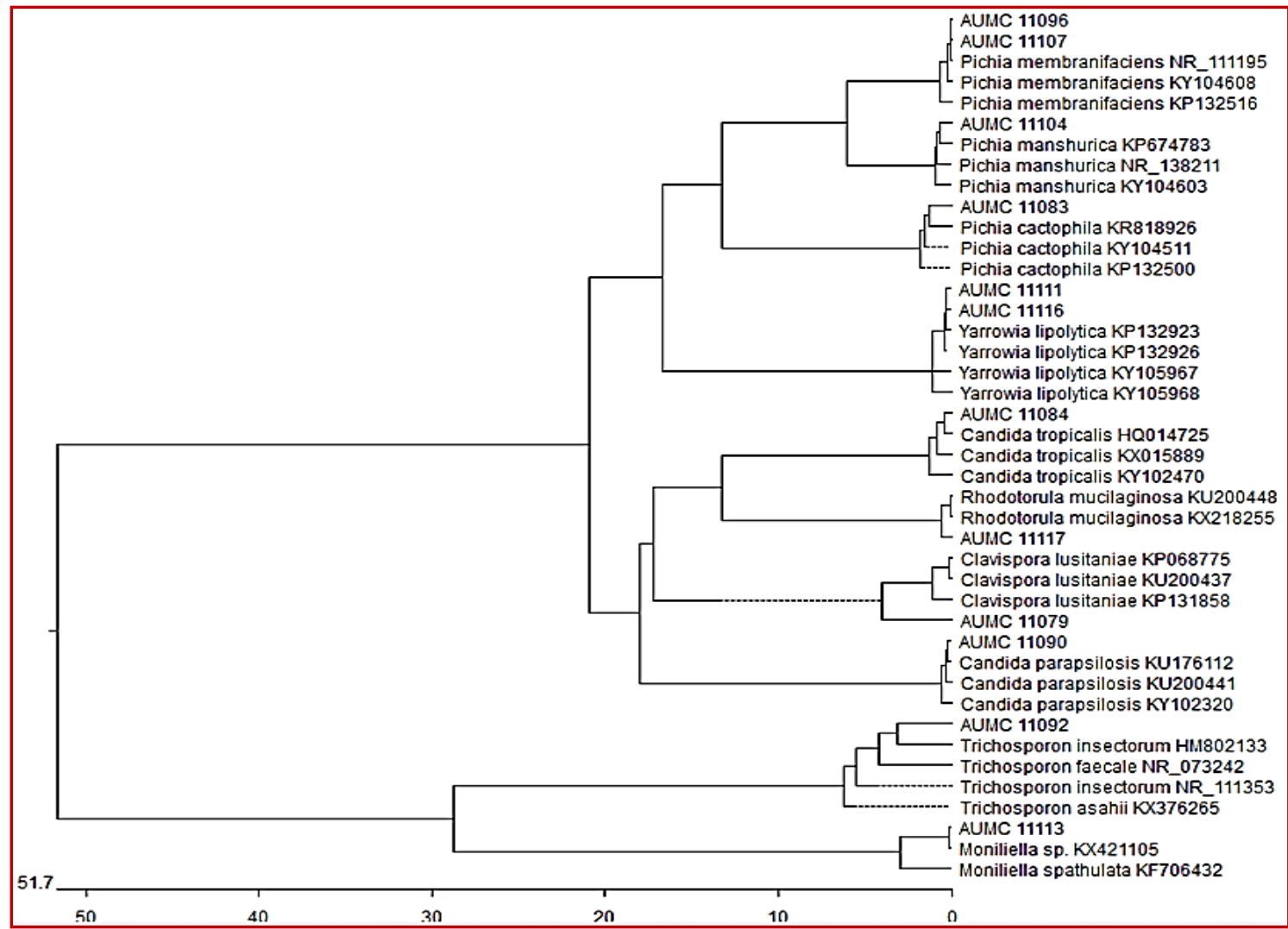

Figure 2. Phylogenetic tree for fungal species isolated from white cheese (given AUMC numbers). Reference strains of corresponding fungi are involved in the tree (given CBS, ATCC or AUMC numbers) 


\subsection{Aflatoxins Contaminating Kareish and Soft Cheese}

The presence of Aflatoxins M1, M2, B1 and B2 in 30 collected samples of kareish (20 samples) and soft cheese (10) was tested using HPLC. Sixteen samples (53.33\%) were contaminated with Aflatoxin M1 (5 and 11 samples of soft cheese and kareish respectively). Aflatoxin M2 was detected only in two samples of kareish cheese as shown in Table 3.

Table 3. Natural occurrence of Aflatoxins in soft cheese samples.

\begin{tabular}{|c|c|c|c|c|c|}
\hline \multirow{2}{*}{$\begin{array}{l}\text { No. of tested } \\
\text { Samples }\end{array}$} & \multirow{2}{*}{$\begin{array}{l}\text { No. of positive } \\
\text { Samples }\end{array}$} & \multicolumn{4}{|c|}{ Aflatoxins (ug/Kg) "ppb" } \\
\hline & & M1 & M2 & B1 & B2 \\
\hline \multirow{5}{*}{10 (Soft cheese) } & \multirow{5}{*}{5} & 1.337 & - & - & - \\
\hline & & 0.670 & - & - & - \\
\hline & & 0.220 & - & - & - \\
\hline & & 0.141 & - & - & - \\
\hline & & 1.425 & - & - & - \\
\hline \multirow{11}{*}{20 (Kareish cheese) } & \multirow{11}{*}{11} & 1.463 & - & - & - \\
\hline & & 0.245 & - & - & - \\
\hline & & 1.219 & 0.073 & - & - \\
\hline & & 1.030 & - & - & - \\
\hline & & 0.606 & - & - & - \\
\hline & & 0.360 & - & - & - \\
\hline & & 0.172 & - & - & - \\
\hline & & 0.250 & - & - & - \\
\hline & & 1.980 & 0.155 & - & - \\
\hline & & 1.827 & - & - & - \\
\hline & & 0.941 & - & - & - \\
\hline
\end{tabular}

The amount of Aflatoxins M1 in positive samples ranged from (0.141-1.980 ug/kg). The highest amount of Aflatoxins M1 was present in kareish cheese while the lowest was in soft cheese samples. All of tested samples were free from Aflatoxins B1 and B2. Levels of AFM1 contamination in kareish cheese ranged from 0.172 to1.980 ug/Kg). Levels of AFM2 in the two positive samples of Kareish were 0.073 and $0.155 \mathrm{ug} / \mathrm{Kg}$. In case of soft cheese, AFM1 was detected in 5 out of 10 samples (50\%), and the contamination level ranged from 0.141 to $1.337 \mathrm{ug} / \mathrm{Kg}$. Contamination of cheese with AFM1may be due to the presence of this toxin in the milk of animals that are fed with aflatoxin B1(AFB1) containing feed [31] or dried milk used in manufacturing cheese [32].

In Egypt, El-Sherief (2000) [33] examined 15 kareish cheese collected from Assiut city, Egyptand found that 2 samples out of 15 kareish cheese tested samples (13.3\%) were contaminated with AFM1 with range 1.75- $3.47 \mathrm{mg} /$ Kg. Gab-Alla et al. (2005) reported that AFM1, AFB1 and mix of AFM1 and AFB1 contaminated 12 samples out of 50 kareish cheese tested samples (24\%) [34]. El-Diasty and Salem (2007) analyzed 20 samples of kareish cheese and found that $20 \%$ of samples were contaminated with AFM1 at a range of 5000 to 3500 ppt [14]. Awad et al. (2014) tested 25 samples of kareish cheese and reported that the mean value of AFM1 was 3600 ppt with contamination percentage of $48 \%$ [35]. El-kest et al. (2015) found that all of the 20 samples of kareish cheese collected from Cairo were contaminated with AFM1 and the average concentration was 140.8 - 528 ppb [36]. In Mansura Governorate, Egypt Younis et al. (2016) evaluated the AFM1 in kareish cheese and observed that $60 \%$ of samples contained the toxin at a concentration of $0.088 \pm 0.024 \mathrm{ppb}$ [37]. El-Sherief (2000) examined 15 soft cheese samples and found that AFM1contaminate (80\%) with the range level of contamination (3.40- 13.75 $\mathrm{mg} / \mathrm{Kg}$ ) [33]. Awad et al. (2014) examined 25 samples of soft cheese for AFM1 contamination and found that mean value was 6700 ppt respectively with contamination percentage $32 \%$ of soft cheese [35]. In Turkey, Var and Kabak (2009) [38], Tekinşen and Uçar (2008) [39] and Ertas et al. (2011) [40] analyzed 20, 100 and 72 cheese samples collected from different cities of turkey and found that $80 \%, 99 \%$ and $94.4 \%$ of cheese samples were contaminated with AFM1 respectively. Also, they reported that the level of AFM1contamination in tested cheese samples were 50-800ng/kg, 0-4100 ng/Kg and 0.012-0.378 mg/Kg respectively. Fallah et al. (2009) [41] and Mariko Kubo (2012) [42] from Iran examined 116 and 80 cheese samples respectively, for AFM1 contamination and found that $80.1 \%$ and $82.5 \%$ of tested cheese samples contaminated with AFM1 respectively. In Brazil, Iha et al. (2011) [43] and Jager et al. (2013) [44] analyzed cheese samples for AFM1 contamination and found that $84 \%$ and $30 \%$ of the samples were respectively contaminated with AFM1. In Kuwait, Dashti et al. (2009) [45] found that $80 \%$ of cheese samples contained AFM1 at levels of 0.024 to $0.425 \mathrm{ng} / \mathrm{Kg}$.

Nearly similar levels of AFM1 contamination of cheese were reported from Sudan (Elzupir and Elhussein, 2010) [46] and Pakistan (Iqbal et al., 2013) [47] where the levels ranged from 79.5- $389 \mathrm{ng} / \mathrm{Kg}$ and $0.091-0.3 \mathrm{mg} / \mathrm{Kg}$ respectively.

On the other hand, Khalifa et al. (2013) analyzed 130 cheese samples for AFM1 contamination and found that, all cheese samples contaminated with Aspergillus spp. were free from AFM1[5].

\section{References}

[1] Pal, M.; Tesfaye S.; Weldegebriel S: b. Hygienic and microbiological aspects of ice cream. Beverage World Food 2012, 39: 42-43.

[2] Foskett D. and Paskins P: The theory of hospitality and catering for levels 3 and 4. London: Hodder for Education 2011.

[3] Osman, O.; Ozturk I.; Bayram O.; Kesmen Z.; Yilmaz M: Characterization of cheese spoiling yeasts and their inhibition by some spices. Egyptian J Dairy Sci 2010, 75(2): 637-640.

[4] Quigley, L.; O'sullivan O.; Stanton C.; Beresford TP.; Ross RP.; Fitzgerald GF.; Cotter PD: The complex microbiota of raw milk. FEMS microbiology reviews 2013, 37(5): 664-698.

[5] Khalifa, M.; Al-Ashmawy M.; Abdel-Khalik A.; El-Sherbini M: Mycological evaluation of serving some dairy products with special reference to mycotoxins production in Azhar University student hostels. World Journal of Dairy \& Food Sciences 2013, 8(2): 165-170.

[6] Hansen, T.; Jakobsen M: Yeast in the dairy industry. Fungal biotechnology in agricultural, food, and environmental applications 2004: 217-231.

[7] Sweeney M. J. and Dobson A. D. W. Mycotoxin production by Aspergillus, Fusarium and Penicillium species. International. Journal of Food Microbiology 1998, 43: 141-158. 
[8] Lopez, C.; Ramos L.; Ramadan S.; Bulacio L.; Perez J: Distribution of aflatoxin M1 in cheese obtained from milk artificially contaminated. International journal of food microbiology 2001, 64(1-2): 211-215.

[9] Pitt J. and Hocking A. Fungi and Food spoilage (3rd Ed.). Springer Science Business Media, LLC, 233 Spring Street, New York, NY 10013. 2009.

[10] Pitt, JI: The genus Penicillium and its teleomorphic states Eupenicillium and Talaromyces. The genus Penicillium and its teleomorphic states Eupenicillium and Talaromyces. 1979.

[11] Leslie J. F. and Summerell B. A. Fusarium laboratory Manual. Blackwell Publishing, John Wiley \& Sons, 338 pp. 2006.

[12] Raper, KB.; Fennell DI: The genus Aspergillus. The genus Aspergillus. 1965.

[13] Zohri, A.; Moharram A.; Refaie R: Mycobiota contaminating beef burger and sausage with reference to their toxins and enzymes. Journal of Basic \& Applied Mycology (Egypt). 2014, 5: 61-73.

[14] El-Diasty, EM.; Salem R: Incidence of lipolytic and proteolytic fungi in some milk products and their public health significance. J Appl Sci Res. 2007, 3(12):1684-1688.

[15] Abd El Goad M.I. Bacteriological and chemical studies on Kareish cheese manufactured from milk at Kalyobia governorate. Journal of the Egyptian Veterinary Medical Association. 68: 141-152. 2008.

[16] Hamms M.A.M., Detection of fungal infection with special reference to their mycotoxin in milk and milk products. $P h D$ Thesis, Dep of Microbiology Faculty of Veterinary Medicine, South Valley University. 2014.

[17] Hameed, KGA: Fungal diversity in different types of cheese and the effect of natamycin on their survival during Feta cheese manufacture and storage. Journal of Advanced Veterinary and Animal Research. 2016, 3(3): 214-220.

[18] Hakim, A.; Azza S.; Rasha M: Isolation, biochemical identification and molecular detection of yeasts from Kareish cheese. Intl J 2013, 4(1): 95-100. Soliman Z.M.S.: Ecological and taxonomical studies on yeasts and other fungi in Egypt,. Dep of Botany and Microbiolog. 2017, Assiut University. 2017.

[19] Soliman N. and Aly S. (2011): Occurrence and identification of yeast species isolated from Egyptian Kareish cheese. Journal of Yeast and Fungal Research. 2 (4): 59-64. 2011.

[20] Soliman Z.M.S.: Ecological and taxonomical studies on yeasts and other fungi in Egypt,. Dep of Botany and Microbiolog 2017, Assiut University. 2017.

[21] Ibrahim, JI.; Salama E.; Saad A.; Helmy AA: Microbial quality of some dairy products in Ismailia city. In: 2nd Conference of Food Safety, Suez Canal University, Faculty of Veterinary Medicine: 2015; 2015: 14-21.

[22] Sayed, M.; Ahmed A.; Shaban W: Microbiological evaluation of some Egyptian white soft cheeses. Benha Vet Med J. 2011, 1:1-6.

[23] ELbagory, A.; Amal ME.; Hammad A.; Salwa A: Prevalence of fungi in locally produced cheese and molecular characterization of isolated toxigenic molds. Benha veterinary medical Journal. 2014, 2:9-20.

[24] Lund, F.; Filtenborg O.; Frisvad J: Associated mycoflora of cheese. Food Microbiology. 1995, 12: 173-180.

[25] Johnson, E.; Cheese products. In E. H. Marth \&. J. L. Steele (Eds.),. Applied dairy microbiology New York: Marcel Dekker 2001. (2nd ed, pp 345-384).

[26] Torkar, KG.; Teger SG: The presence of some pathogen micro organisms, yeasts and moulds in cheese samples produced at small dairy-processing plants. Acta Agric Slov 2006, 88: 37-51.

[27] Lavoie, K.; Touchette M.; St-Gelais D.; Labrie S: Characterization of the fungal microflora in raw milk and specialty cheeses of the province of Quebec. Dairy science \& technology. 2012, 92(5): 455-468.
[28] Allaraj Y., AÇ, Biba N., Spahiu1 M. and Cuka E.,: Preliminary data on mycotic evaluation in cheeses sold at Tirana markets. International journal of ecosystems and ecology science (IJEES). 3: 113-118. 2013.

[29] Banjara, N.; Suhr MJ.; Hallen-Adams HE: Diversity of yeast and mold species from a variety of cheese types. Current microbiology. 2015, 70(6): 792-800.

[30] Chipilev, N.; Daskalov H.; Stoyanchev T: Study on the prevalence of lipolytic yeasts and moulds in raw cow milk and white brined cheese. Bulgarian Journal of Veterinary Medicine. 2016, 19(2).

[31] Van Egmond H: Mycotoxins. In: Residues and contaminants in milk and milk products. International Dairy Federation 1991 (Special Issue 9101): 131-145.

[32] Blanco, jl.; Domínguez 1.; Gómezlucía e.; Garayzabal jf.; Goyache j.; Suárez g: Behavior of aflatoxin during the manufacture, ripening and storage of Manchego-type cheese. Journal of Food Science. 1988, 53(5): 1373-1388.

[33] El-Sherief, L: Incidence of mycoflora and some mycotoxins in locally manufactured cheese. MV Sc. Thesis, Fac. Vet. Med., Assiut Univ; 2000.

[34] Gab-Alla H.M., SEDWM, and Abdel Fattah M.E.: Mycological Evaluation of some types of cheese. uez canal veterinary medicine journal, 2005 VIII(2): p 1-8. 2005.

[35] Awad, EI.; Mansour IHAH.; Ismail S-EY: Qualitative and Quantitative Detection of Aflatoxin M1 Residues in White Soft Cheese. Alexandria Journal of Veterinary Sciences. 2014, 40(1): 119-123.

[36] El-kest, MM.; El-Hariri M.; Khafaga N.; Refai MK: Studies on Contamination of Dairy Products by Aflatoxin M1 and Its Control by Probiotics. J Glob Biosci. 2015, 4(1): 1294-1312.

[37] Younis, G.; Ibrahim D.; Awad A.; El Bardisy M: Determination of aflatoxin M1 and ochratoxin A in milk and dairy products in supermarkets located in Mansoura City, Egypt. Adv Anim Vet Sci. 2016, 4(2): 114-121.

[38] Var, I.; Kabak B: Detection of aflatoxin M1 in milk and dairy products consumed in Adana, Turkey. International Journal of Dairy Technology. 2009, 62(1): 15-18.

[39] Tekinşen, KK.; Uçar G: Aflatoxin M1 levels in butter and cream cheese consumed in Turkey. Food Control. 2008, 19(1): 27-30.

[40] Ertas, N.; Gonulalan Z.; Yildirim Y.; Karadal F: A survey of concentration of aflatoxin M1 in dairy products marketed in Turkey. Food Control. 2011, 22(12): 1956-1959.

[41] Fallah, AA.; Jafari T.; Fallah A.; Rahnama M: Determination of aflatoxin M1 levels in Iranian white and cream cheese. Food and chemical toxicology. 2009, 47(8): 1872-1875.

[42] Kubo, M: Mycotoxins Legislation Worldwide. Leatherhead Food Research, Randalls Road, Leatherhead, Surrey KT22 7RY UK. 2012: 1-6.

[43] Iha, MH.; Barbosa CB.; Okada IA.; Trucksess MW: Occurrence of aflatoxin M1 in dairy products in Brazil. Food Control. 2011, 22(12): 1971-1974.

[44] Jager, A.; Tedesco M.; Souto P.; Oliveira C: Assessment of aflatoxin intake in São Paulo, Brazil. Food Control. 2013, 33(1): 87-92.

[45] Dashti, B.; Al-Hamli S.; Alomirah H.; Al-Zenki S.; Abbas AB.; Sawaya W: Levels of aflatoxin M1 in milk, cheese consumed in Kuwait and occurrence of total aflatoxin in local and imported animal feed. Food Control. 2009, 20(7): 686-690.

[46] Elzupir, AO.; Elhussein AM: Determination of aflatoxin M1 in dairy cattle milk in Khartoum State, Sudan. Food Control. 2010, 21(6): 945-946.

[47] Iqbal, SZ.; Asi MR.; Jinap S: Variation of aflatoxin M1 contamination in milk and milk products collected during winter and summer seasons. Food control. 2013, 34(2): 714-718. 